\title{
A Mechanical Vibration-induced Electric Energy Generation (MVEG) and Applications to Ride Quality of Vehicles and International Roughness Index (IRI)
}

\author{
Schun T. Uechi ${ }^{1}$, Hiroshi Uechi ${ }^{2}$ \\ ${ }^{1}$ Nomura Research Institute (NRI), Secure Tech., Ltd., Irvine, CA 92614, USA \\ ${ }^{2}$ Osaka Gakuin University, Suita, Osaka 564-8511, Japan \\ Correspondence: Schun T. Uechi, Nomura Research Institute (NRI), Secure Tech., Ltd., Irvine, CA 92614, USA.
}

Received: April 23, 2019 Accepted: May 27, 2019 Online Published: May 28, 2019

doi:10.11114/set.v6i1.4301 URL: https://doi.org/10.11114/set.v6i1.4301

\begin{abstract}
A mechanical vibration-induced, electric energy harvesting method is discussed with applications to vibration analyses of systems of vehicles, motorboats, trains, machines and bridges, etc.. The research has evolved from the analysis of International Roughness Index (IRI), which studies roughness of road-surface as longitudinal vibrational motions in a vehicle measured with a quarter-car simulation (QCS) or Global Positioning System (GPS) with sensors such as gyro sensor and magnetometer sensor. The electric energy-convertible vibrations with information of roughness of road surface are extracted by way of an mechanoelectric energy conversion, and an energy harvesting technology suitable for the system of vehicles is discussed. The mechanical vibration-induced electric current is also suitable for IRI information measurement as well as a measure for ride quality of vehicles.
\end{abstract}

Keywords: Information and energy harvesting, Simulations of mechanical vibration-induced electric cur-rent, International roughness index (IRI), Ride quality (Rq)

\section{Introduction}

The vibrations of mechanical systems and machines, such as vibrations of floor and wall, buildings and bridges, vehicles, railway trains or tracks, are often considered useless for creating unwanted sounds and waste of energies, and so, careful designs to minimize unwanted vibrations and methods to prevent, absorb and damp undesirable vibrations have been developed. These vibrations are random or irregular like the movement on a gravel rough road with external impacts and forces, and they appear as a complicated superposition of many kinds of oscillations. This is the reason why vibration phenomena are unwanted and metaphorically considered as trash and waste in the first place.

An oscillation is characterized by its own frequency or energy-spectrum directly connected to energy of the oscillation, and vibrations are mixture of all energy states. If vibrations are understood as dynamical phenomena, trash and waste can be regarded as useful resource of materials and energy to be recycled. However, trash and waste must be conveniently separated and partitioned with corresponding energy extracting technological devices, and hence, energy-convertible oscillations are recognized useful if corresponding energy-extracting devices are available.

Technologies designed to improve energy efficiency, sustainable and renewable energy sources, such as solar, wind and wave, thermal and electromechanical energy are actively investigated to reduce negative human impacts on natural environment and ecosystems. The engineering field of technology to find and collect energies from solar and luminous energy, waves and oscillations, heat and current of particles is known as energy-harvesting technology, which is expected to be sustainable sources of energy (Priya S. \& Inman J. D.; 2009, Wang X. 2016). The purpose of energy harvesting technologies is to provide power sources, energy-storage devices or transmission of converted power to electrical grids in general. These technologies are considered to reduce the green-house gas emissions for sustainability of natural environment.

The current research has developed from the analysis of mechanical vibrations of vehicles responding to roughness of road surface, resulting in constructing the profile of road-surface, known as International Roughness Index (IRI) (Uechi T. S. \& Uechi H. 2018; Du Y, Liu C, Wu D \& Jiang S. 2014; Kropac O. \& Mucka P. 2009). The road roughness and surface-bump measurements have been applied for restoring and repairing roads which are undermined by longtime use 
of roads or earthquakes, and it is expected to keep road quality (Reina G, Leanza A \& Messina A. 2018; Kasaiezadeh A, Jahromi R. M. \& Alasty A. 2005). The profile of IRI is a collection of big-data for roughness of road surface and hence, it demands computationally intensive tasks and time for analyses, primarily by employing statistical method to obtain probabilistic results. It is not readily accessible for all researchers to check data because it demands certain budget of research and takes much computation time to get a probabilistic result, which restricts accessibility to the data and impedes progresses of the field. The roughness of road surface is one of random phenomena, but vibrations induced in a system should be understood as dynamical phenomena. It is necessary to introduce a method to understand corresponding results given by statistical big-data and associated dynamical vibration phenomena.

We proposed a dynamical model by introducing a model Lagrangian to analyze mechanical systems of vibrations and the profile of IRI (Uechi T. S. \& Uechi H. 2018)., which would help the analysis of vibrations induced by impacts of rough road surfaces. The Lagrangian model of vibrations can be applied to the analysis of unmanned surface vehicles (USV), or wave energy converter (WEC), related to dynamical motions of three-dimensional systems affected by internal oscillations and trembles. In the current paper, a mechanoelectric energy conversion from vibrations to electric energy and its application to the profile of IRI and ride-quality are discussed.

Mechanical vibrations of vehicles are usually diminished by sophisticated modern suspension technologies by way of tires, tire air, springs, shock absorbers. The mechanical system of vehicles can be considered as the multi-leveled, stratified spring systems as shown in Fig. 1. Drivers and passengers are on the higher level of the system whose ride quality is maximized and vibrations of vehicles are minimized, and unwanted vibrations and impacts are absorbed and maintained in the low-level spring system. This is essential for the concept of mechanical-induced electric energy conversion by employing an flexible energy-converter module simplistically drawn as a black box z3 in Fig. 2, which is shown in sec. 2. However, because the energy-converter module, z3, is different from conventional converter designs, technical details are not disclosed due to the preparation of patent applications.

The energy harvesting technologies and designs have been developing, and demands for the fabrication of the efficient electronics are actively investigated by employing piezoelectric-based energy harvesting devices of crystals and ceramics which can generate a small voltage whenever they are mechanically deformed. The piezoelectric effect is the internal generation of electrical charge resulting from an applied mechanical force, and the reverse piezoelectric effect is the internal generation of a mechanical strain resulting from an applied electrical field. The energy harvesting and storage systems of intermediate and macroscopic energy harvesting technologies are essential for applications, recycles of energies and sustainability of ecology and natural environment. The technological design, construction, and maintenance of the natural and artificial environment are disciplines of the civil engineering, which encompasses mechanics of physics, materials science and electrical circuitry, and hence, researchers from vast fields of sciences and engineering would contribute to energy harvesting research. There are many vibrational, thermodynamical, wind and wave, solar and luminous, heat and chemical phenomena able to be applied to energy harvesting technologies.

Mechanical vibrations have both information and energy produced by corresponding systems, but these useful vibrations are extracted when an appropriate energy-conversion module is realized. The energy conversion-module used for numerical calculations can be directly applied to other mechanical systems of vibrations. In section 2, energy-convertible damping oscillations obtained from mechanical systems are discussed. The numerical results of energy-convertible oscillations and induced electric charge and current are shown in section 3. The applications to IRI and ride quality are discussed in section 4, and conclusions and perspectives are in section 5.

\section{Energy-Convertible Mechanical Vibrations in Systems of Vehicles}

Mathematical models of vehicle's response-type measurement for roughness of road surface have been investigated since 1940s starting from applications to airplanes and military vehicles. Mechanical responses to external impacts are essential for aircrafts moving runways, standard fabrication for steel of fast-growing railroads, maintenance of infrastructure of road and automobiles (The National Cooperative Highway Re-search Program (NCHRP) 1962). The vibrations caused by road surfaces, vehicles, buildings, bridges and railways are important information on mechanical structures and materials.

Because of the demand of ride quality at the upper level of vehicles supported by suspension systems, the lower suspension systems are expected to absorb most of vibrations and impacts exerted on a vehicle. Although the suspension model in Fig. 1 seems to be crudely simplified, we discussed that it can reasonably reproduce realistic vibrational motions experienced in our daily life, and then, we numerically extracted independent damping oscillations produced by external impacts exerted by roughness of road surface (Uechi T. S. \& Uechi H. 2018).

The equations of motion for vibrations associated with the profile of IRI data are discussed with the

Lagrangian, $L_{\mathrm{IRI}}$, resulting in (Uechi T. S. \& Uechi H. 2018): 


$$
\begin{gathered}
m_{1} \ddot{z}_{1}+C_{1} \dot{z}_{1}-k_{1}\left\{\left(z_{1 G}-z_{1}\right)-\left(z_{2 c}-z_{2}\right)\right\}=0, \\
m_{2} \ddot{z}_{2}+C_{2} \dot{z}_{2}+k_{1}\left\{\left(z_{1 G}-z_{1}\right)-\left(z_{2 G}-z_{2}\right)\right\}-k_{2}\left(z_{2 G}-z_{2}\right)=-k_{2}\left(z_{2}-X(t)\right),
\end{gathered}
$$

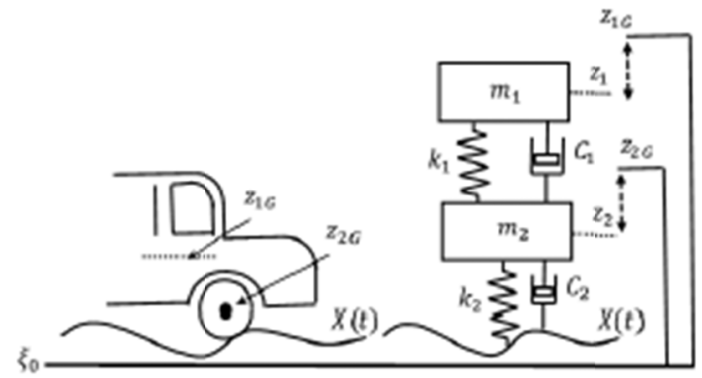

Figure 1. The example of simplified two-dimensional, two-stratified spring system

with the center of mass expression, $z_{1 G}$ and $z_{2 G}$. The values of masses, $m_{1}, m_{2}$, and centers of masses, $z_{1 G}=0.50(\mathrm{~m}), z_{2 G}=$ $0.30(\mathrm{~m})$, are fixed, and the variables are defined from variations of centers of masses as, $\Delta z_{1}(t)=z_{1}(t)-z_{1 G}$ and $\Delta z_{2}(t)=$ $z_{2}(t)-z_{2 G}$, respectively . The spring constants, $k_{1}, k_{2}$, and phenomenological dissipation constants, $C_{1}, C_{2}$, are optimally adjusted to obtain oscillation-induced electric current in numerical simulations. The roughness of road-surface function, $X(t)$, denotes longitudinal changes in time for a road surface, and the speed of vehicles is supposed to be a walking speed, for instance, of a two-wheeled car (Segway), or at most about $10 \mathrm{~km} / \mathrm{h}$, indicating that tires of a vehicle should be full-contact on the road surface.

One should note that initial conditions of $z_{1}(0)$ and $z_{2}(0)$ with the external, surface function $X(t)=0$ (a flat, smooth road surface) result in constants in time: $z_{1}(t)=z_{1 G}$ and $z_{2}(t)=z_{2 G}$. The surface function, $X(t)$, must be a piecewise continuous function of time as emphasized in the reference (Uechi T. S. \& Uechi H. 2018), which is essential to extract and determine independent, energy-convertible oscillations. The possible energy-convertible oscillations are found as responses to roughness of a road surface, starting and breaking a vehicle, and other three-dimensional shear and torsional motions could be expected for energy-convertible oscillations.

Based on the discussion reproducing the profile of IRI, we obtained a collection of energy-convertible damping oscillations in the low level spring system (see, the coupled $m_{2}$ in Fig. 1). The energy-conversion module, $z_{3}$, is attached in $m_{2}$ as in Fig. 2 of the stratified spring system, and energy-convertible oscillations are extracted in the module $z_{3}$ which has a specific design for vibration-induced energy conversion module for the system.

The oscillating module $z_{3}$ is a mechanoelectric energy-converting system which has a small magnet of mass $m_{3} m_{1}, m_{2}$ with springs, electromagnetic coils and capacitors. The Lagrangian of the mechanical vibration sector is given by,

$$
\mathcal{L}=\mathcal{L}_{\text {IR }}+\frac{1}{2} m_{3} \dot{z}_{3}(t)^{2}+k_{3}\left(z_{3}(t)-z_{3 G}\right)^{2}+\mathcal{L}_{\mathrm{m}-\varepsilon},
$$

where the spring $z_{3}(t)$ is assumed to be balanced at the center of oscillation, which is defined as $z_{3 G} \equiv 0$ for numerical calculations and $L_{\mathrm{m}}$ is the Lagrangian of mechanoelectric energy conversion. As explained in Introduction, technical details of energy-converter module are not disclosed due to the preparation of patent applications. The phenomenological power function is assumed as $C_{3} z_{3}{ }^{2} / 2$, and the response to external force is coupled from $z_{2}$ to $z_{3}$ as $-C_{4}\left\{\left(z_{2 G}-z_{2}(t)\right)-\right.$ $X(t)\} \theta(t),\left(\theta(t)\right.$ is a step function). The equation of motion for $z_{3}(t)$ is therefore given by,

$$
m_{3} \ddot{z}_{3}(t)+C_{3} \dot{z}_{3}(t)+2 k_{3} z_{3}(t)=-C_{4}\left\{\left(z_{2 G}-z_{2}(t)\right)-X(t)\right\} \theta(t),
$$

and initial conditions are $z_{3}(0)=0$ and $z_{3}{ }_{3}(0)=0$. It should be noted that when an external force on $z_{3}(t)$ is initially zero, the solution to the eq. (2.4) is only $z_{3}(t)=0$ for all $t \geq 0$. However after the impacts of force from roughness of road surface or trembles, starting and breaking or changes of acceleration are received, smooth damping oscillation solutions will be produced in $z_{3}$; for example, the numerical simulations for responses to the road-surface function, $X(t)$, are shown with the following four external forces of impacts (Uechi T. S. \& Uechi H. 2018): 


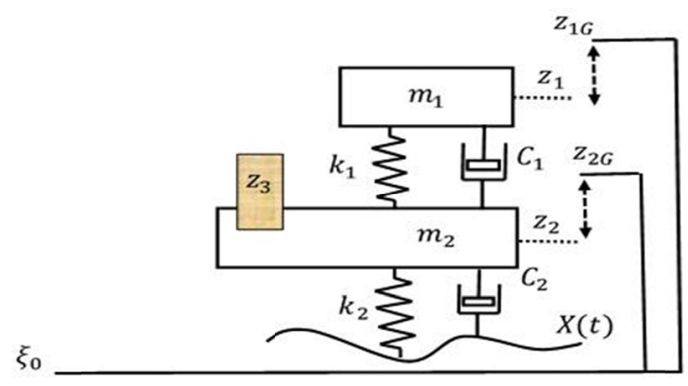

Figure 2. The oscillation converter module $z_{3}$ in the strati ed mechanical vibration system

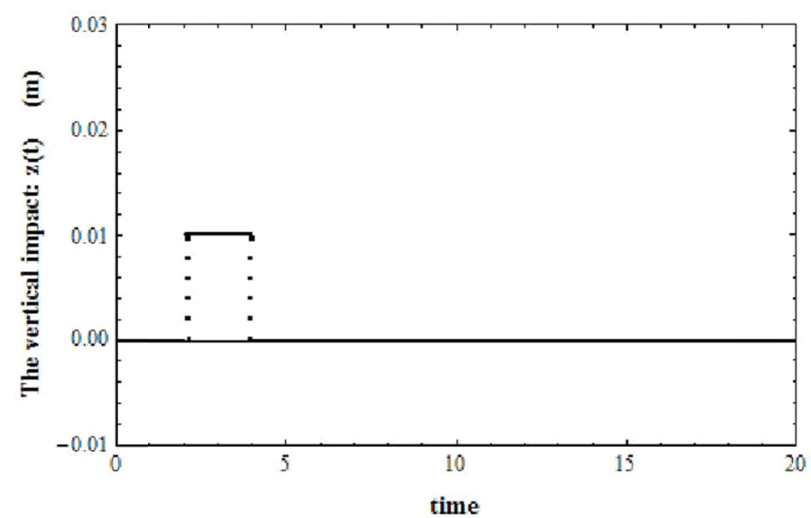

(a) The one-convex rectangular impact

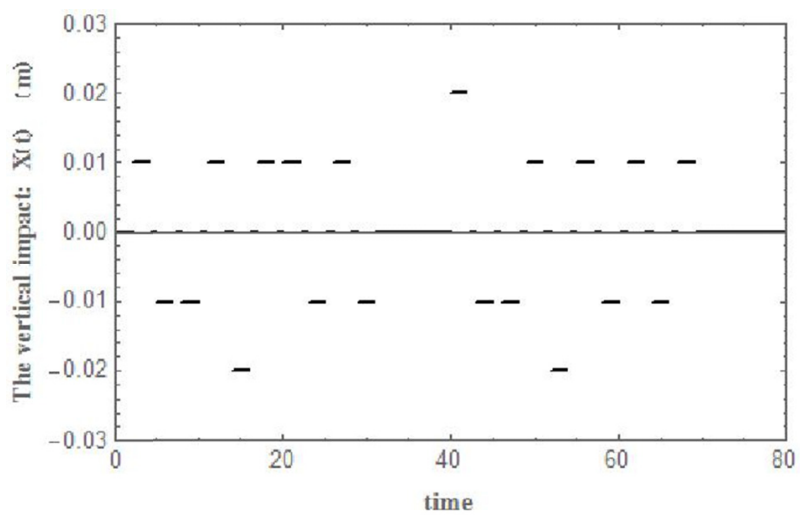

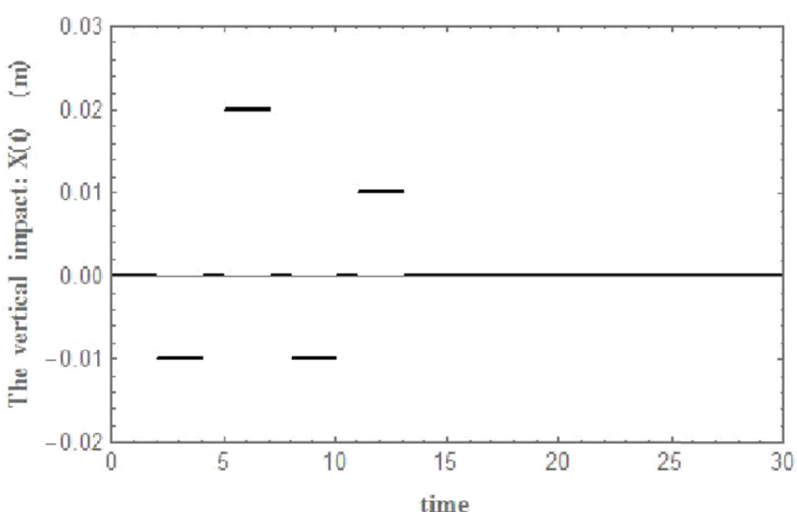

(b) The four convex-concave rectangular impacts

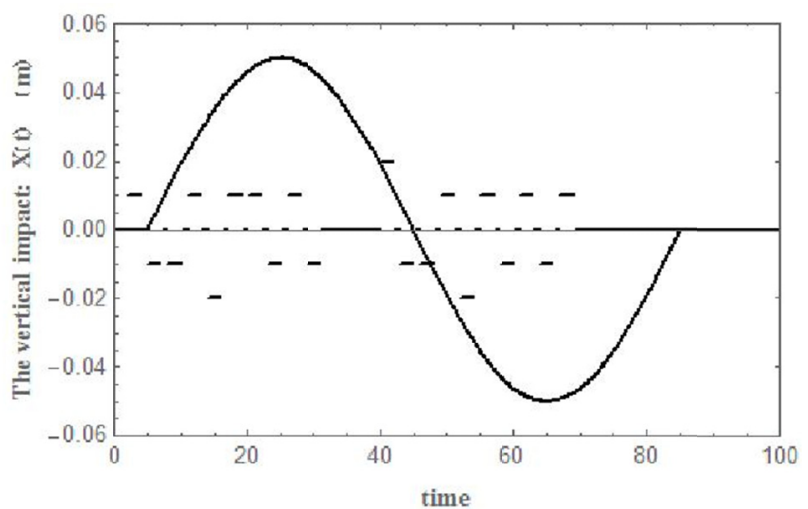

(c) The 10-cluster +10 -cluster convex-concave rectangular impacts (d) The convex-concave impacts with smooth upward and downward slopes

Figure 3. The examples of roughness of road surface. The external forces of impacts, (a) (d), are used to generate electric charge and current. They are also used to generate corresponding associated roughness index (ARI) (Uechi T. S. \& Uechi H. 2018)

1. $X(t)$ : A rectangular convex impact (the vertical height of impact: $\mathrm{h}_{i}=1.0 \mathrm{~cm}$ ).

2. $X(t)$ : The four convex-concave rectangular impacts.

3. $X(t)$ : The 10-cluster +10 -cluster convex-concave rectangular impacts.

4. $X(t)$ : The convex-concave impacts with smooth upward and downward slopes. The crest $(5.0 \mathrm{~cm})$ and trough $(-5.0$ $\mathrm{cm}$ ) are supposed to reach in 20 seconds and then, 40 seconds walking-speed, respectively.

The rectangular external forces or impacts exerted by the rough road surfaces, $X(t)$, are respectively shown in Fig. 3, and the vertical height of impacts within $h_{i}= \pm 1.0 \pm 2.0 \mathrm{~cm}$ is utilized. The impacts are randomly chosen for rough road-surface simulations. They generate variations of $z_{2}(t): \Delta z_{2}(t)=z_{2}(t)-z_{2 G}$, coupled to the spring $z_{3}(t)$ vibrations. 
The stratified spring system generates smooth damping oscillations convertible to electricity that can be found in the module of $z_{3}$ where a technical scheme for mechanical vibration-based electromagnetic generation is specifically designed, and the module $z_{3}$ is removable and flexibly installed in other vibrational systems for oscillation analyses. We will discuss solutions to equations from (2.1) (2.4) and suggest the current results of mechanical vibration-induced electric current as a standard measure for the profile of international roughness index (IRI) (Uechi T. S. \& Uechi H. 2018; Du Y, Liu C, Wu D \& Jiang S. 2014; Kropac O. \& Mucka P. 2009) and ride quality of vehicles (Reina G, Leanza A \& Messina A. 2018; Kasaiezadeh A, Jahromi R. M. \& Alasty A. 2005).

\section{The Mechanical Vibrations and Induced Charges and Currents}

The numerical simulations of four external forces of impacts, $X(t)$, in Fig. 3 are employed to show mechanical vibrations, $z_{2}(t)$ and $z_{3}(t)$, the induced electric charge $Q(t)$ and current $I(t)$. The strength of rectangular external force of impacts is within, $\mathrm{h}_{i}=1.02 .0 \mathrm{~cm}$, and each impact continues 2.0 seconds in time. Though forces of impacts seem to be rough at the first glance from the figures, the longitudinal impacts of road surface 3(a) 3(d) are relatively smooth in practice, because the speed of vehicles is supposed to be about $10 \mathrm{~km} / \mathrm{h}$. Phenomenological constants in eqs. (2.1) (2.4) are adjusted and fixed in all calculations so that the characteristic responses of oscillations, $z_{2}(t)$ and $z_{3}(t)$, and the induced currents $I(t)$ can be clearly observed. The model constants connected to roughness of road surface are also numerically tested for simulations of IRI (Uechi T. S. \& Uechi H. 2018). The coupling constants are fixed in the current numerical calculation as:

$m_{1}=300 \mathrm{~kg}, m_{2}=0.25 m_{1}, m_{3}=10{ }^{5} m_{1} ; k_{1}=1.5 m_{1}, k_{2}=0.25 m 1, k_{3}=1.5 m_{3} ; C_{1}=7.0 m_{1}, C_{2}=$ $0.05 m_{1}, C_{3}=0.20 m_{3}, C_{4}=0.20 m_{3}$.

These coupling constants should be determined in practice by experiments of ride quality of vehicles and optimal designs of energy-conversion of the module $z_{3}$.

\subsection{The Simulation 1: X(T), A Rectangular Convex Impact, the Induced Charge and Current (Fig. 4)}

The external force of impact 3(a) produces the vibration $\Delta z_{2}(t)$, and simultaneously, the energy convertible mechanical oscillation, $z_{3}(t)$, is produced. The mechanoelectrical energy conversion module $z_{3}$ produces the associated charge $Q(t)$ and electric current $I(t)$, shown in figures $4(\mathrm{c})$ and $4(\mathrm{~d})$, respectively. The roughness of road surface defined by a rectangular convex impact $\left(\mathrm{h}_{i}=1.0 \mathrm{~cm}\right)$ produces the damping oscillation, $\Delta z_{2}(t) .0 .3(\mathrm{~cm})$ in Fig. $4(\mathrm{a})$, simultaneously generating the oscillation-damping, $z_{3}(t)$, with the maximum amplitude $\left|z_{3}{ }^{\max }\right| 0.15 \mathrm{~cm}$.

The induced charge, $Q(t)$, in a capacitor in the converter module is a smooth oscillation-damping function of time with the maximum magnitude, $\left|Q^{\max }\right| 0.2 \mathrm{mC}$. The induced current, $I(t)$, is a smooth oscillation-damping function in time with the maximum current, $\left|I^{\max }\right| 0.4 \mathrm{~mA}$, but the discrete changes of positions, $\Delta z_{2}(t)$ or $z_{3}(t)$, by the external force of impact become more visible in the induced electric current shown in Fig. 4(d), because the electric current is given by the derivative of charge as $I(t)=d Q(t) / d t$. The electric current is susceptible to discrete changes of oscillations in $z_{3}$, and it is suitable for detecting discrete, longitudinal mechanical vibrations. Conventional and piezoelectric energy converters (Arroyoa E., Badela A., Formosaa F., Y. Wu Y \& J. Qiu J. 2012; Constantinou P, Mellor PH \& Wilcox PD. 2012) may be applicable for the design of the energy-conversion module.

The phenomenological dissipation constants, $C_{1}, C_{2}, C_{3}, C_{4}$, and spring constants, $k_{1}, k_{2}, k_{3}$ determine responses to external forces and overall dynamical motions of the coupled system. When these constants are increased, reductions and restrictions to frequency and amplitude of oscillations are observed, resulting in an rapid oscillation damping, and with some combinations of constants, one often obtains no oscillatory solutions which are irrelevant to mechanoelectric energy conversions. The ratios among phenomenological constants are important for determining dynamical motions of a coupled spring system, but the overall property of simulations is not sensitive to the change of mass, $m_{1}$, as long as it is large compared to other component masses. The motions of other component masses, $m_{2}$ and $m_{3}$ in the current model, are sensitive to ratios of phenomenological constants, which is also empirically observed in coupled mechanical systems.

\subsection{The Simulation 2: X(T), the Four Convex-Concave Rectangular Impacts, the Induced Charge and Current (Fig. 5)}

The external four convex-concave impacts $3(\mathrm{~b}),\left(\left|h_{i}\right|=1.0\right.$ or $\left.2.0 \mathrm{~cm}\right)$, produce the associated vibrations, $\Delta z_{2}(t)$, and the coupled energy-convertible mechanical oscillation, $z_{3}(t)$. Accordingly, the mechanical oscillation, $z_{3}(t)$, generates the electric charge $Q(t)$ and current $I(t)$ as shown in Figs. 5(c) and 5(d). The maximum amplitude of $\Delta z_{2}{ }^{{ }^{2 a x}}(t) .0 .6(\mathrm{~cm})$ in Fig. $5(\mathrm{a})$, and the oscillation-damping is generated in $z_{3}(t)$ with the maximum amplitude $\left|z_{3}{ }^{\max }\right| 0.30 \mathrm{~cm}$.

The induced charge $Q(t)$ by the four convex-concave rectangular impacts in Fig. 3(b) shows a smooth damping oscillation with $\left|Q^{\max }\right| 0.60 \mathrm{mC}$. The induced current $I(t)$ also shows an oscillation-damping property with $\left|I^{\max }\right| 1.5 \mathrm{~mA}$, and the timing of impacts coming from roughness of the road surface can be observed as discrete longitudinal changes in the electric current in Fig. 5(d). The roughness of road surface can be precisely observed as discrete changes in the mechanical vibration-induced current $I(t)$. 

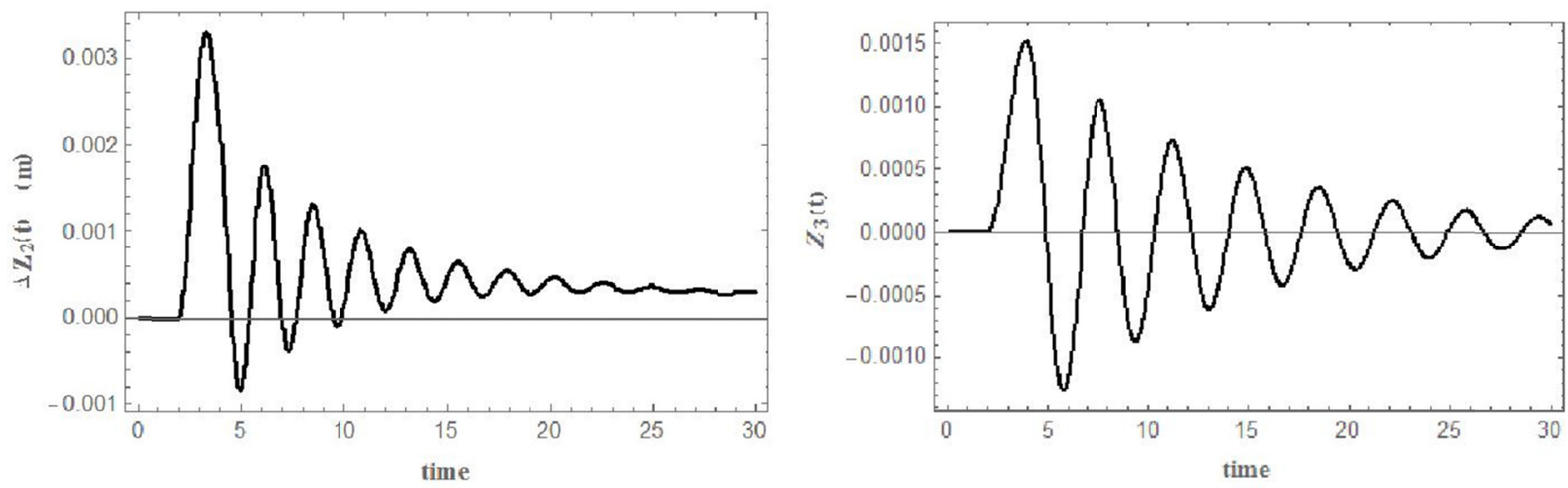

(a) The associated variation of position, $\Delta z_{2}(t)=z_{2}(t)-z_{2 G}(\mathrm{~m})$. (b)The variation of the spring, $z_{3}(t)(\mathrm{m})$ in the energy-conversion module

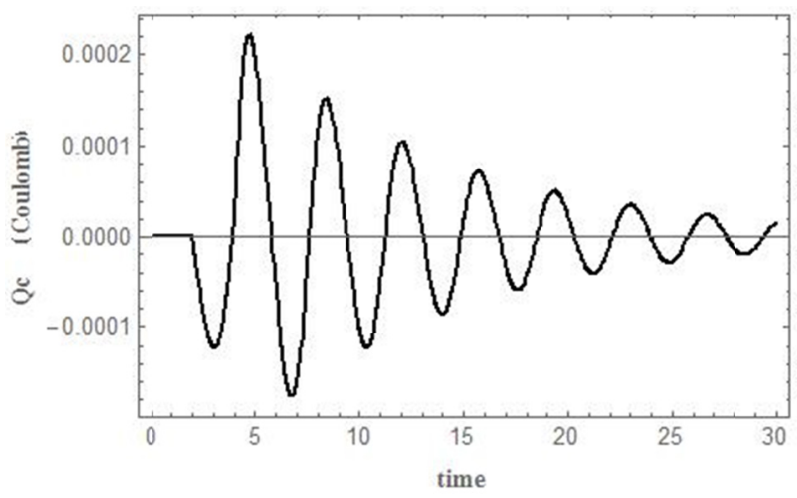

(c) The variation of the induced charge, $Q(t)$

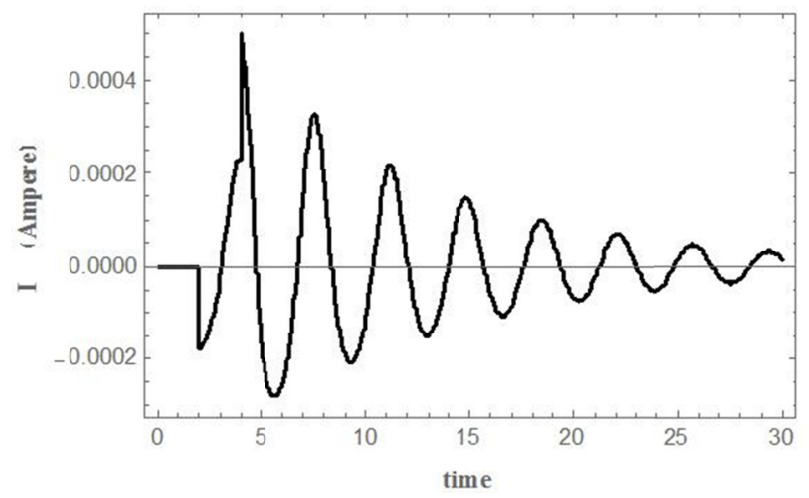

(d) The associated electric current: I(t) (amp)

Figure 4. The reaction to one-convex rectangular impact: $X(t)$, Fig. 3(a). $\Delta z 2(t)=z 2(t)-z_{2 G}$ and the variation of $z_{3}(t)$, the induced charge $Q(t)$ and electric current $I(t)$

3.3 The Simulation 3: X(T), the 10-Cluster + 10-Cluster Convex-Concave Rectangular Impacts, the Induced Charge and Current (Fig. 6)

The 10-cluster +10 -cluster convex-concave rectangular impact 3(c) produces the associated vibration, $\Delta z_{2}(t)$, and the coupled energy-convertible mechanical oscillation, $z_{3}(t)$ in Figs. 6(a) and 6(b). The rough-ness of road surface 10-cluster +10 -cluster convex-concave rectangular impacts $\left(\left|h_{i}\right|=1.02 .0 \mathrm{~cm}\right)$ is arbitrarily introduced as shown in Fig. 3(c) for numerical simulations. The induced charge $Q(t)$ and electric current $I(t)$ are respectively shown in Figs. 6(c) and 6(d).

The longitudinal motions, $\Delta z_{2}(t)$ with the maximum amplitude $\Delta z_{2}{ }^{\max } 0.6(\mathrm{~cm})$, and $z_{3}(t)$ with the maximum amplitude $\left|z_{3}^{\max }\right| 0.30(\mathrm{~cm})$ are produced in Figs. 6(a) and 6(b). The induced charge $Q(t)$ in Fig. 6(c) shows a complex but continuous oscillation with $\left|Q^{\max }\right| 0.40 \mathrm{mC}$. The associated current $I(t)$ also shows a corresponding complex oscillation with $\left|I^{\max }\right| 1.0$ $\mathrm{mA}$ and discrete longitudinal motions in Fig. 6(d).

There is no problem to convert mechanical vibration energy to electric energy, even if the response of longitudinal oscillations becomes extremely intensive as roughness of a road surface becomes complicated, and it seems that information on roughness of road surface would not be tractable. However, discrete changes coming from complicated bumps of road surface and responses in the induced current $I(t)$ can be tractable clearly by changing time-range of data; for example, by changing time-range from $0 \leq t \leq 80$ to $0 \leq t \leq 20$, analogous to magnifying data-response in a specific time-range. The property is different from the big-data of IRI which could be in any background of uncorrelated random frequencies known as white noise. The center of position and induced current, $\Delta z_{2}(t)$ and $I(t)$, are very useful together to study roughness of road surface and the profile of IRI. 


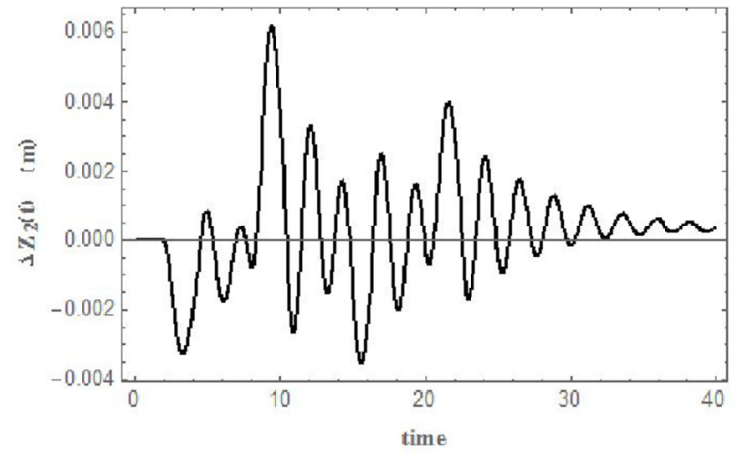

(a) The associated variation of position, $\Delta z_{2}(t)=z_{2}(t)-z_{2 G}(\mathrm{~m})$

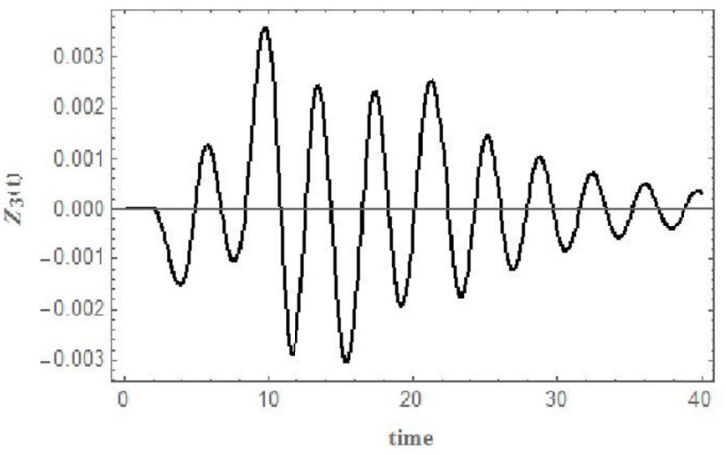

(b) The variation of the spring, $z_{3}(t)(\mathrm{m})$.

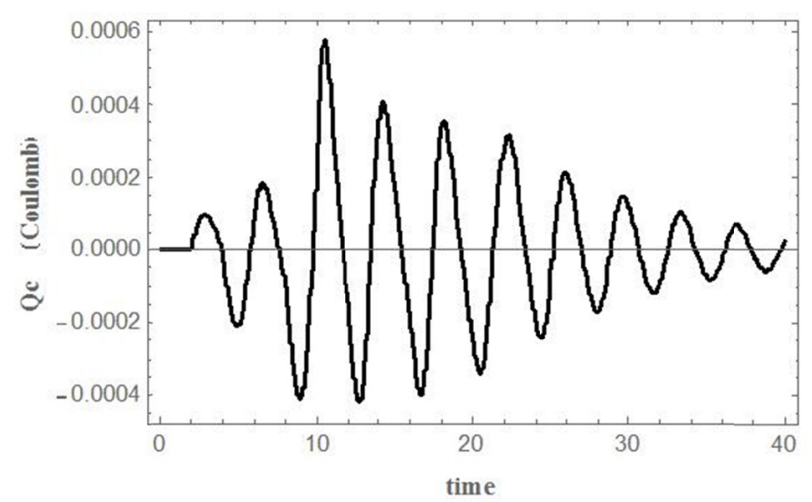

(c) The variation of the induced charge, $Q(t)(\mathrm{C})$

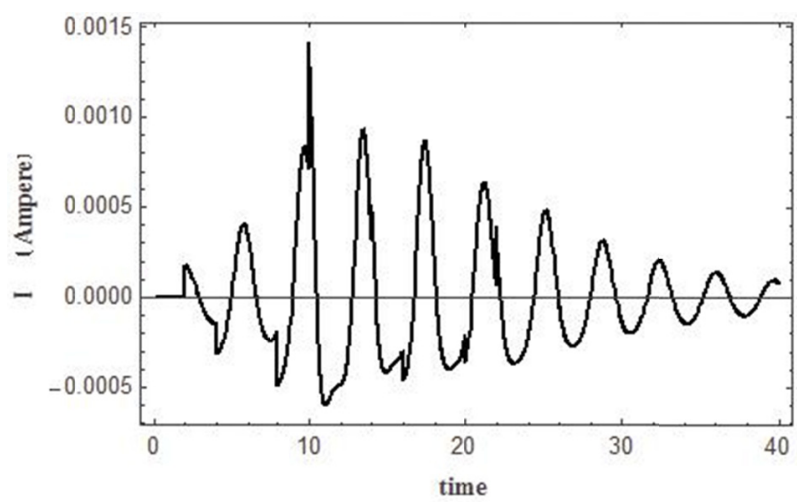

(d) The associated electric current: I(t) (amp)

Figure 5. The reaction to the four convex-concave rectangular impacts $X(t)$ : Fig. $3(\mathrm{~b}) . \Delta z_{2}(t)=z_{2}(t)-z_{2 G}$, the variation of the spring, $z_{3}(t)$, the induced charge $Q(t)$ and electric current $I(t)$

3.4 The Simulation 4: X(T), the Convex-Concave Impacts With Smooth Upward and Downward Slopes, the Induced Charge And Current (Fig. 7)

The 10-cluster +10 -cluster convex-concave rectangular impact with smooth upward and downward slopes 3(d) is employed for the numerical simulation, resulting in the associated vibration, $\Delta z_{2}(t)$ and the coupled energy-convertible mechanical oscillation, $z_{3}(t)$, shown in Figs. 7(a) and 7(b), respectively. The roughness of road surface, 10-cluster + 10-cluster convex-concave rectangular impacts, is combined with smooth upward and downward slopes, which are separately shown in Fig. 3(d). The crest $(5.0 \mathrm{~cm})$ and trough $(-5.0 \mathrm{~cm})$ of the slope are supposed to reach in 20 seconds and then, 40 seconds walking-speed, respectively. The induced electric charge $Q(t)$ and electric current $I(t)$ are shown in Figs. $7(\mathrm{c})$ and $7(\mathrm{~d})$. The vibration of $\Delta z_{2}(t) .4 .0(\mathrm{~cm})$ in Fig. 7(a) simultaneously generates oscillations, $z_{3}(t)$, with the maximum amplitude $\left|z_{3}{ }^{\max }\right| 1.0 \mathrm{~cm}$. The induced charge $Q(t)$ in Fig. 7(c) shows a complex but smooth oscillation with $\left|Q^{\max }\right| 3.0 \mathrm{mC}$. The associated current $I(t)$ also shows corresponding complex oscillations with $\left|I^{\max }\right| 8.0 \mathrm{~mA}$., but the force of external impacts shown in Fig. 3(d) can be readily observed in Fig. 7(d) by changing time-range of observation as explained in the simulation 3. 


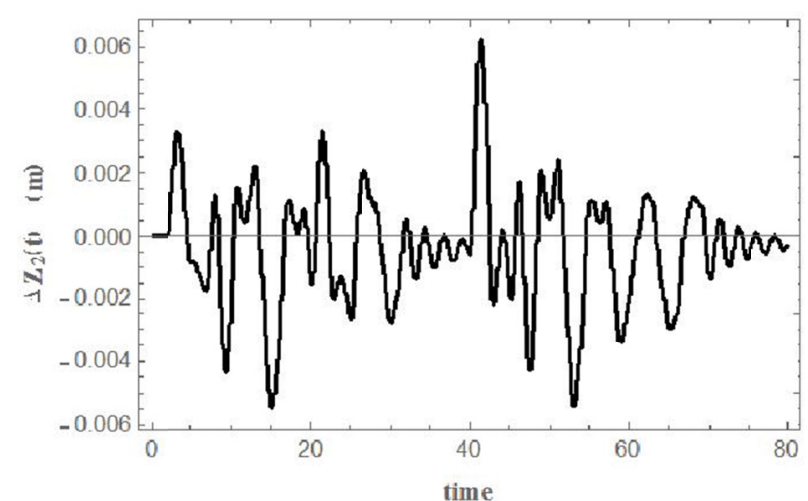

(a) The associated variation of position, $\Delta z_{2}(t)=z_{2}(t)-z_{2 G}(\mathrm{~m})$

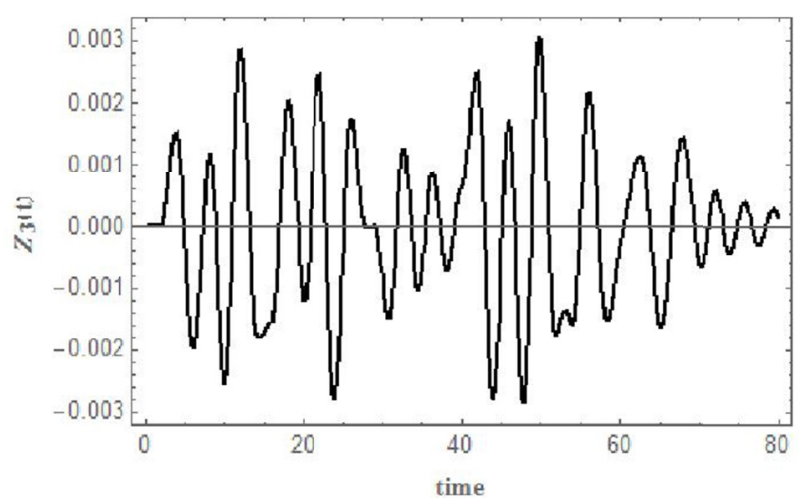

(b) The variation of the spring, $z_{3}(t)(\mathrm{m})$

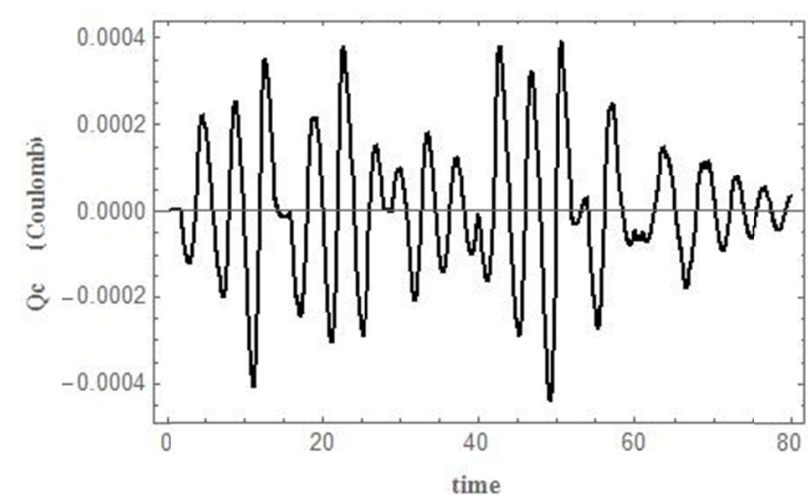

(c) The variation of the induced charge, $Q(t)(\mathrm{C})$

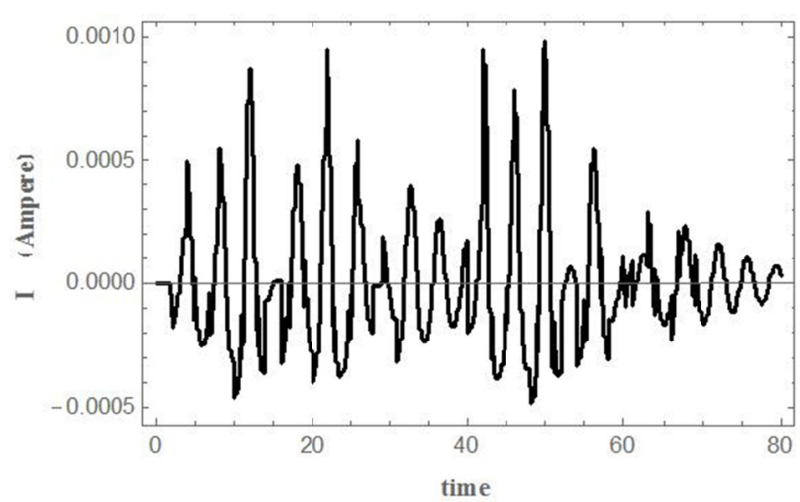

(d) The associated electric current: $\mathrm{I}(\mathrm{t})(\mathrm{amp})$

Figure 6 . The reaction to the 10 -cluster +10 -cluster convex-concave rectangular impacts, $X(t)$ : Fig. $3(\mathrm{c}) . \Delta z_{2}(t)=z_{2}(t)-z_{2 G}$ and the variation of the spring, $z_{3}(t)$, the induced charge $Q(t)$ and electric current $\mathrm{I}(t)$

The response of longitudinal oscillations becomes complicated with characteristic upward and downward shifts in $z_{2}(t)$ and $z_{3}(t)$, and so, it is useful to examine physical responses and correspondence between longitudinal oscillations and roughness of road surface from $z_{2}(t)$ and $z_{3}(t)$. Even if complicated discrete changes of road surface are introduced, timing of discrete impacts can be checked with the induced current $I(t)$. The electric current $I(t)$ and position data $\Delta z_{2}(t)$ are useful together to examine responses to roughness of road surface and used as complementary information to complete the profile of IRI. 


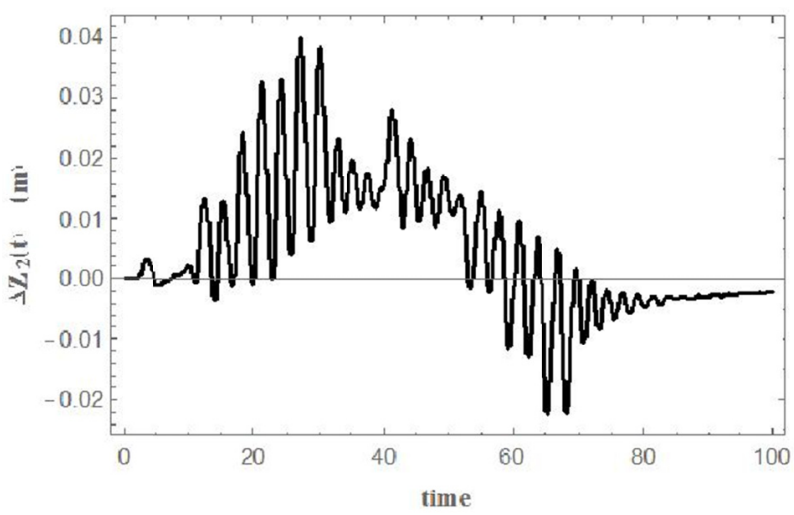

(a) The associated variation of position, $\Delta z_{2}(t)=z_{2}(t)-z_{2 G}(\mathrm{~m})$

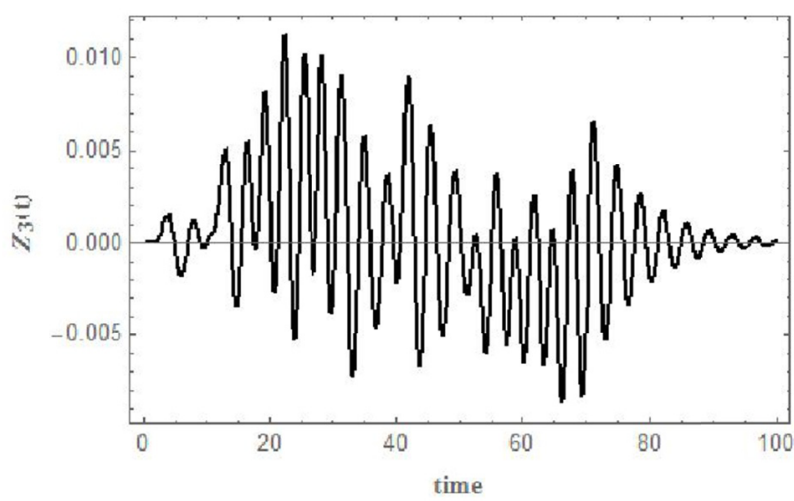

(b) The variation of the spring, $z_{3}(t)(\mathrm{m})$.

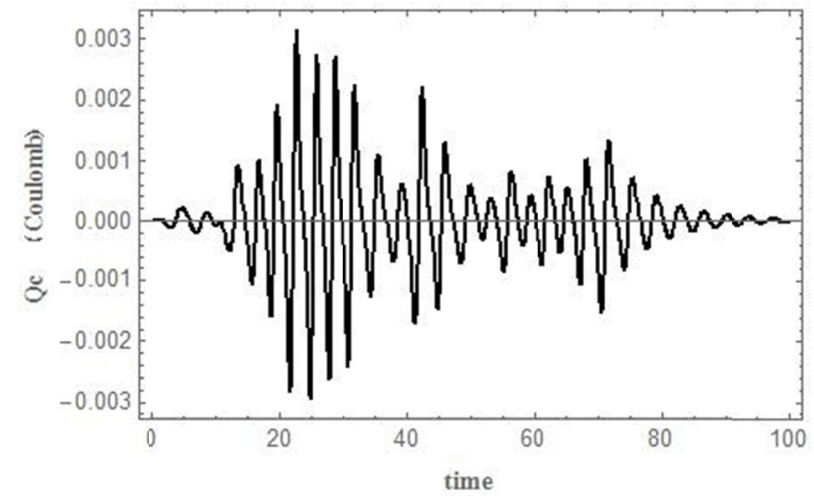

(c) The variation of the induced charge, $Q(t)(\mathrm{C})$

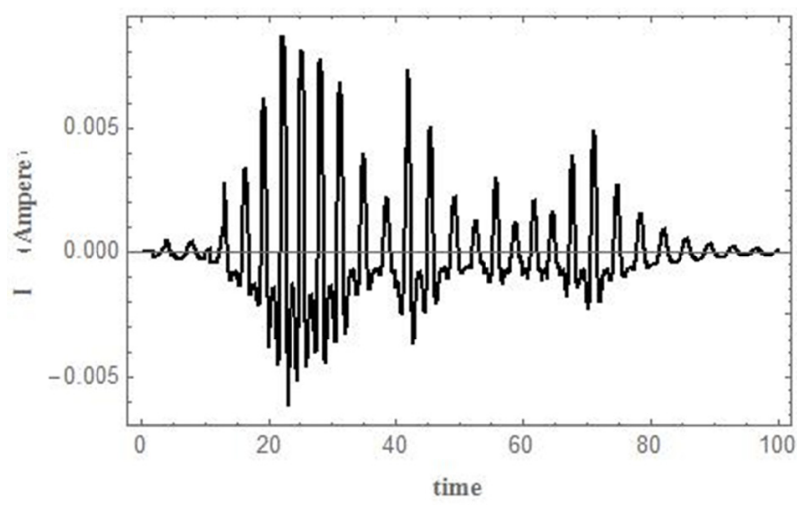

(d) The associated electric current: I(t) (amp)

Figure 7. The reaction to the 10-cluster +10 -cluster convex-concave rectangular impacts with smooth up-ward and downward slopes, $X(t)$ : Fig. 3(d). $\Delta z_{2}(t)=z_{2}(t)-z_{2 G}$, the variation of the spring, $z_{3}(t)$, the induced charge $Q(t)$ and electric current $I(t)$

\section{The Applications of Energy Harvesting Technology to IRI and Ride Quality, Rq}

The modern technology employs elaborated sensors to measure high-speed longitudinal motions, resulting in time series of big-data expressed as compressed longitudinal spikes of data. Because the time series of longitudinal spikes of data obtained from modern censors include background noise, statistical analysis is used in general in order to extract information of road surface. The useful concepts and relations among the big-data of IRI and mechanical impacts of road-roughness function, associated roughness index (ARI), acceleration and position are studied by the Lagrangian, $L_{\text {IRI }}$ (Uechi T. S. \& Uechi H. 2018). In the current paper, we have shown characteristic vibrations of mechanical oscillations and vibration-induced charge and current by employing the Lagrangian model and discussed the mechanical vibration-induced electric current $I(t)$ which has important information on roughness of road surface and compensates for completing the profile of IRI data.

The vibration analysis is applied to detect abnormal conditions, defects of machine and a mechanical system in order to reduce maintenance cost and risks of engineering system failure (Eshleman R. 1999). A mechanical system needs to keep reliability for a specified period of time and energy efficiency, and the analysis of a vibration spectrum provides important frequency information that can pinpoint defects at a component of the system. An energy harvesting technology can be used to extract energy as well as information of a system, signals of defects, ride quality and IRI, only if such an appropriate device or module is realized. We have shown in the current paper that the vibrational energy and information on a mechanical system can be extracted; energy and information can be extracted simultaneously with an appropriate energy-harvesting technology.

It is commonly known that modern vehicle's effectiveness in insulating drivers and passengers from, vibrations bumps or corrugations on a road surface has been studied actively, which is known as ride quality. The research field of ride quality is related to suspension components which connect frames of a vehicle to wheels, such as springs, shock absorbers and 
bushings for reducing vibrations. Hence, a calibration of roughness index of a road-surface is closely related to ride quality. A profile of road surface corresponding to impacts of external force, bumps and slopes as well as ride quality will be studied in detail by the current theoretical model, because the module $z_{3}$ can be removed and flexibly installed to higher spring systems or to other part of a coupled component, and the numerical simulations can be shown as explained in the sec. 3 .

For example, let us examine $\Delta z_{1}(t)=z_{1}(t)-z_{1 G}$ and $\Delta z_{2}(t)=z_{2}(t)-z_{2 G}$ induced by the four convex-concave rectangular impact 3(b) and consider a possible definition of ride quality. One could define intensity of a vibration in certain time range $t_{0} \leq t \leq t_{1}$ as:

$$
A=\int_{t_{0}}^{t_{1}}\left|Z_{1}(t)\right|^{2} d t \quad \text { and } \quad B=\int_{t_{0}}^{t_{1}}\left|Z_{2}(t)\right|^{2} d t .
$$

Ride quality may be defined by the ratio: $R_{q}=A / B$, and if $R_{q}$ is much less than 1 , it means that ride quality of the upper spring-system of a vehicle is excellent and unwanted vibrations are almost absorbed in a lower layer of the suspension system. The results are shown in Fig. 8, and in the current spring system, vibrations of $z_{1}$ are clearly diminished compared to those of $z_{2}$. The index of ride quality in the current example is numerically given by, $R_{q} 0.104$ in the time range $\left(t_{0}=0 \leq\right.$ $t \leq t_{1}=40$ ). We suggest that researchers who have real corresponding vibration data of vehicles examine the applicability of ride quality index, $R_{q}$.

The relation between the energy harvesting technologies and information on mechanical vibration systems is an interesting discovery, and these fields would help each other progress supporting and enhancing its own technology and reliability.

\section{Conclusions and Perspectives}

Mechanical vibration-induced electric energy generation (MVEG) and applications to ride quality of vehicles and International Roughness Index (IRI) are an interesting technological field to study. The definition and quantitative analyses of ride quality can be possible by employing mechanical vibration-induced electric current shown specifically in the paper. The vibrations of mechanical systems are essentially useful physical phenomena in terms of information of system and energy. The concept has been developed by modern technological advancement of small, autonomous electronic devices and accumulators for energy conversion, harvesting and storage systems from external sources, such as solar, thermal, wind and wave, osmotic processes (Kathy L. 2014). The potential technological applications to above mentioned fields by way of piezoelectric and new materials (Alhawari M., Mohammad B., Saleh H. \& Ismail M. 2018), as well as an electromagnetic induction method might lead to enormous applications with the demand of necessity, accessibility and availability invoked from all humane social activities.
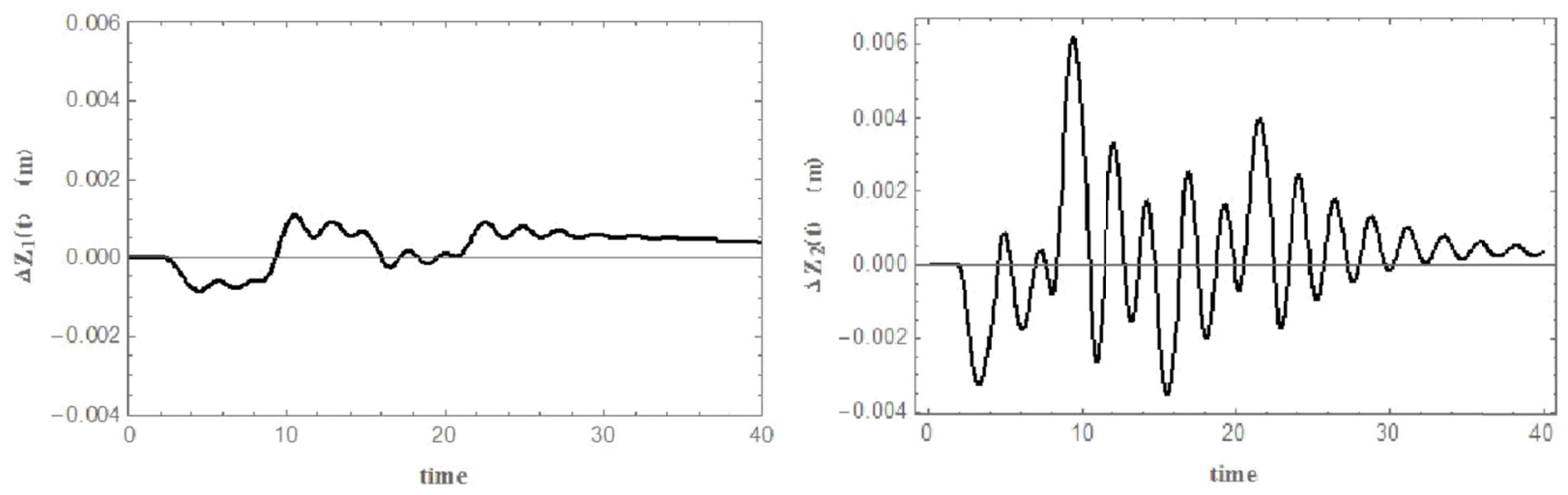

(a) The response of $\Delta z_{1}$ to the four external convex- concave rectangular impacts (b) The response of $\Delta z_{2}$ to the same rectangular impacts

Figure 8 . The example for ride quality of a vehicle responding to the four external convex-concave rectangular impacts of 3(b). The vibrations of $\Delta z_{1}$ are diminished and smoother than those of $\Delta z_{2}$

The energy-convertible mechanical oscillations are discussed and found with associated energy harvesting technologies, and the advancement of extraction, storage and management of harvesting technology will expand the scope of innovations, theoretical foundations and testability. The microelectronic technology or nanogenerator could have a promising impact on technological applications as well as theoretical foundations of science as manifestations of physical laws of mechanics, thermodynamics, electromagnetism and quantum physics, and the profound elaboration of theoretical foundations could be expected, resulting mutual development in theoretical and technological progresses. The energy 
generation, transducer and storage technologies for small, wireless autonomous devices used in wearable electronics and wireless sensor networks are expected to become reliable power modules, and more importantly, we need sustainable power stations for large systems in the near future (Bowen R. C., Topolov Y. V. \& Kim A. H. 2016); for that purpose, convenient and interesting, useful energy harvesting technologies should be developed and investigated further.

\section{References}

Alhawari, M., Mohammad, B., Saleh, H., \& Ismail, M. (2018). Energy Harvesting for Self-Powered Wearable Devices, Springer. https://doi.org/10.1007/978-3-319-62578-2

Arroyoa, E., Badela, A., Formosaa, F., Wu, Y., \& Qiu, J. (2012) Comparison of electromagnetic and piezoelectric vibration energy harvesters: model and experiments. Sensors Actuators A 183, 148-156. https://doi.org/10.1016/j.sna.2012.04.033

Bowen, R. C., Topolov, Y. V., \& Kim, A. H. (2016). Modern Piezoelectric Energy-Harvesting Materials, Springer. https://doi.org/10.1007/978-3-319-29143-7

Constantinou, P., Mellor, P. H., \& Wilcox, P. D. (2012). A magnetically sprung generator for energy harvesting applications. Mechatronics, IEEE/ASME Trans, 17(3), 415-424. https://doi.org/10.1109/TMECH.2012.2188834

Du, Y., Liu, C., Wu, D., \& Jiang, S. (2014). Measurement of International Roughness Index by Using Z-Axis Accelerometers and GPS. Mathematical Problems in Engineering. https://doi.org/10.1155/2014/928980

Eshleman, R. (1999). Basic machinery vibrations: An introduction to machine testing, analysis, and monitoring.

Kasaiezadeh, A., Jahromi, R. M., \& Alasty, A. (2005). Fatigue Life Assessment Approach to Ride Comfort Optimization of a Passenger Car under Random Road Execution Conditions, SAE International by University of Edinburgh. https://doi.org/10.4271/2005-01-0805

Kathy, L. (2014). Materials in Energy Conversion, Harvesting, and Storage, John Wiley \& Sons.

Kropac, O., \& Mucka P. (2009). Classification Scheme for Random Longitudinal Road Unevenness Considering Road Waviness and Vehicle Response. Shock and Vibration, 16, 273-289. https://doi.org/10.1155/2009/935858

Priya, S., \& Inman, J. D. (2009). Energy Harvesting Technologies, Springer. https://doi.org/10.1007/978-0-387-76464-1

Reina, G., Leanza, A., \& Messina, A. (2018). On the vibration analysis of off-road vehicles: Influence of terrain deformation and irregularity, Journal of Vibration and Control 1-19. https://doi.org/10.1177/1077546318754682

The National Cooperative Highway Research Program (NCHRP). (Established in 1962). Federal Highway Administration and US Department of Transportation.

Uechi, T. S., \& Uechi, H. (2018). The Profiling of International Roughness Index (IRI) Based on Lagrangian Method. World Journal of Engineering and Technology, 6(2018), 885-902. https://doi.org/10.4236/wjet.2018.64059

Wang, X. (2016). Frequency Analysis of Vibration Energy Harvesting Systems, Elsevier. https://doi.org/10.1016/B978-0-12-802321-1.00009-1

\section{Copyrights}

Copyright for this article is retained by the author(s), with first publication rights granted to the journal.

This is an open-access article distributed under the terms and conditions of the Creative Commons Attribution license which permits unrestricted use, distribution, and reproduction in any medium, provided the original work is properly cited. 